\title{
Evaluating unspecific oxidative stress parameters in the sera of patients with irritable bowel syndrome
}

\author{
EMEL YIGIT KARAKAS ${ }^{1}$ \\ TIMUCIN AYDOGAN ${ }^{2}$ \\ HATICE SEZEN ${ }^{3}$ \\ TURGAY ULAS ${ }^{1}$ \\ ${ }^{1}$ Harran University, Faculty of Medicine \\ Department of Internal Medicine, Sanliurfa/Turkey \\ ${ }^{2}$ Harran University School of Medicine \\ Department of Gastroenterology, Sanliurfa/Turkey \\ ${ }^{3}$ Harran University School of Medicine \\ Department of Biochemistry, Sanliurfa/Turkey
}

\section{Correspondence:}

Assoc. Prof. Dr. Turgay Ulas

Harran University School of Medicine

Department of Internal Medicine

Yenisehir Campus, 63000, Sanliurfa/Turkey

Email: turgayulas@yahoo.com

Keywords: Irritable bowel syndrome, total oxidant status, total antioxidant status, oxidative stress index

Received September 19, 2015.

Revised March 24, 2016.

Accepted April 26, 2016.

\begin{abstract}
Objective: Irritable bowel syndrome (IBS) is a common global condition characterized by abdominal pain and alterations in bowel habits not caused by other organic diseases and its etiopathogenesis has not been elucidated. In this study, we aimed to evaluate the oxidative stress parameters in patients with IBS.

Materials and methods: Fifty patients diagnosed with IBS using the Rome III criteria and a control group of 50 healthy subjects were included in the study. Oxidative stress parameters including total oxidant status (TOS), total antioxidant status (TAS) and oxidative stress index (OSI) values were analyzed from all study subjects.

Results: Compared to the controls; the TOS and OSI values were significantly higher, and the TAS value was significantly lower in IBS patients $(p<0.001$ for all $)$.
\end{abstract}

Conclusions: In present study we demonstrated that oxidative stress increased and antioxidant capacity decreased in IBS, and antioxidants might be beneficial in the supportive treatment for IBS.

\section{INTRODUCTION}

Tritable bowel syndrome (IBS) is a common global condition that impairs quality of life. It is characterized by abdominal pain and altered bowel habits not caused by other known organic diseases. Abdominal pain in IBS is usually described as a crampy sensation with variable intensity and periodic exacerbations. The location and character of the pain can vary widely. Emotional stress and eating may exacerbate the pain, while defecation often provides some relief $(1,2)$. Altered bowel habits are ranging from diarrhea and constipation or normal bowel habits alternating with either diarrhea and/or constipation. Constipation may last from days to months, with interludes of diarrhea or normal bowel function. Stools are often hard and patients may also experience a sense of incomplete evacuation even when the rectum is empty. Diarrhea is usually occurs during waking hours, most often in the morning or after meals and accompanied by lower abdominal cramps and urgency. Stools generally characterized as frequent loose stools of small to moderate volume with mucus discharging. Numerous factors have been implicated in the etiology of IBS, including genetics, intestinal infections, over-production of intestinal bacteria, increased cytokine response and inflammation, irregularity of serotonergic functions, and psychosocial aspects (3-8). Since it has been regarded as a functional bowel disease because of the etiopathogenesis of IBS has not 
been fully illuminated, diagnosis of IBS is made based on the Rome III clinical criteria, last modified in 2006 (9).

The term oxidative stress is used to describe a series of chemical reactions that result in the production of free oxygen radicals and other reactive molecules (10). These free radicals and reactive oxygen molecules are neutralized by the complex structure of the antioxidant system but when the pro-oxidant and antioxidants systems are dysregulated, oxidative stress can damage important cellular components including lipids, proteins and nucleic acids $(11,12)$. It was already showed that the oxidative stress products increase if there is an endocrine disease that affects the body's metabolic rate and an inflammatory disease which cause acute or chronic organ damage in previous studies (13-17). But there is limited number of studies investigating the presence of oxidative stress in non organic and functional diseases. Therefore the current study was undertaken and aimed to evaluate the role of oxidative stress in IBS etiopathogenesis by measuring the total oxidative status (TOS), total antioxidant status (TAS) values and calculating oxidative stress index (OSI) by Erel's method which is easy, stable, reliable, sensitive, inexpensive and fully automated in IBS patients.

\section{MATERIALS AND METHODS}

The study participants included 50 patients who presented at the Gastroenterology Polyclinic of Harran University Medical Faculty and were diagnosed with IBS, and a control group of 50 healthy individuals. The diagnosis of IBS was made according to the Rome III criteria9. Routine laboratory studies (complete blood count, fasting blood glucose, urea, creatinine, alanine amino transferase, aspartate amino transferase, alkaline phosphatase, gama glutamil transferase, sedimentation, C- reactive protein, thyroid stimulating hormone) and abdominal ultrasonographic examination were evaluated while selection of patients to exclude from the other diseases. Patients $<18$ years old, addicted to alcohol or medication, and those with a previous diagnosis of hematological, renal, hepatic, cardiovascular, neurological, chroninc inflammatory bowel disease, chronic inflammatory connective tissue diseases and malign diseases were excluded from the study. Informed consent, conforming to the Helsinki Declaration of 2008, was obtained from all patients and control subjects. The local ethics committee approved the study protocol.

\section{Biochemical Analysis}

All blood samples were drawn from a large antecubital vein without interruption of venous flow, following an overnight fasting state into blood tubes and immediately stored on ice at $4^{\circ} \mathrm{C}$. The serum was then separated from the cells by centrifugation at $3000 \mathrm{rpm}$ for $10 \mathrm{~min}$ and they were stored until analyzing at $-80{ }^{\circ} \mathrm{C}$. Ten milliliters of blood was used for baseline routine laboratory tests. Thyroid stimulatin hormone (TSH), levels were analyzed using an electrochemiluminescence immunometric assay

Table 1: Baseline comparison of the demographic, laboratory and clinical characteristics of all subjects

\begin{tabular}{|lccc|}
\hline Parameters & IBS Group $(\mathrm{n}=50)$ & Healthy subjects $(\mathrm{n}=50)$ & $\mathrm{p}$ \\
\hline Age (years) & $37.2 \pm 11.9$ & $39.5 \pm 11.7$ & 0.932 \\
Sex (male/female) & $27 / 23$ & $28 / 22$ & 0.989 \\
Hematocrit (\%) & $41.0 \pm 4.6$ & $41.7 \pm 4.3$ & 0.646 \\
Glucose (mg/dL) & $99.2 \pm 7.1$ & $93.9 \pm 4.3$ & 0.277 \\
Urea (mmol/L) & $28.5 \pm 8.9$ & $28.0 \pm 10.8$ & 0.587 \\
Creatinine (mg/dL) & $0.6 \pm 0.1$ & $0.7 \pm 0.1$ & 0.534 \\
AST (U/L) & $24.3 \pm 11.6$ & $23.9 \pm 14.2$ & 0.219 \\
ALT (U/L) & $27.5 \pm 19.4$ & $29.4 \pm 25.3$ & 0.761 \\
TSH (mIU/L) & $2.7 \pm 0.7$ & $2.5 \pm 0.8$ & 0.511 \\
\hline
\end{tabular}

Abbreviations: IBS: Irritable Bowel Syndrome, AST: aspartate aminotransferase, ALT: alanine aminotransferase, TSH: Thyroid stimulating hormone

Table 2: Mean \pm SD of TAS, TOS and OSI levels

\begin{tabular}{|lccc|}
\hline Parameters & IBS Group $(\mathrm{n}=50)$ & Healthy subjects $(\mathrm{n}=50)$ & $\mathrm{p}$ \\
\hline TAS $(\mu \mathrm{mol}$ Trolox equivalent/L) & $850 \pm 80.0$ & $1040 \pm 90.0$ & $<0.001$ \\
TOS $\left(\mu \mathrm{mol} \mathrm{H} \mathrm{O}_{2}\right.$ equivalent/L) & $44.84 \pm 1.56$ & $37.87 \pm 1.11$ & $<0.001$ \\
OSI (arbitrary units) & $5.27 \pm 0.48$ & $3.64 \pm 0.26$ & $<0.001$ \\
\hline
\end{tabular}

Abbreviations: IBS: Irritable Bowel Syndrome, TAS: Total Antioxidant Status, TOS: Total Oxidant Status, OSI: Oxidative Stress Index 
(ECLIA) method with a Roche Elecsys E170 immunoanalyzer (Roche Diagnostics, Burgess Hill, UK). Serum urea, creatinine (Crea), fasting blood glucose (FBG), aspartate aminotransferase (AST), alanine aminotransferase (ALT), were determined using commercially available assay kits (Abbott ${ }^{\oplus}$, Abbott Park, North Chicago, Illinois, USA) with an auto-analyzer (Abbott ${ }^{\circledR}$, Abbott Park, North Chicago, Illinois, USA).

\section{Measurement of Total Oxidant Status}

Serum TOS was measured using a novel automated method developed by Erel (18). Oxidants present in the sample oxidize the ferrous ion-odianisidine complex to ferric ion. The oxidation reaction is enhanced by glycerol molecules, which are abundant in the reaction medium. The ferric ion generates a colored complex with Xylenol Orange in an acidic medium. Color intensity, which can be measured spectrophotometrically (V-530; Jasco ${ }^{\oplus}$, Tokyo, Japan), is related to the quantity of oxidant molecules present in the sample. The assay is calibrated with hydrogen peroxide and the results expressed in terms of micromolar hydrogen peroxide equivalents per liter $(\mu \mathrm{mol}$ $\mathrm{H}_{2} \mathrm{O}_{2}$ equivalent/L).

\section{Measurement of Total Antioxidant Status}

Serum TAS was measured using a novel automated method developed by Erel (19). In this method, hydroxyl radical, the most potent biological radical, is produced. In the assay, ferrous ion solution in reagent 1 is mixed with hydrogen peroxide present in reagent 2 . Sequentially-produced radicals, such as the brown-colored dianisidinyl radicalcation produced by the hydroxyl radical, are also potent radicals. This method allows measuring the antioxidant effect of the sample against potent freeradical reactions that are initiated by the hydroxyl radical. The assay has excellent precision values of more than 97\%. The results are expressed as $\mu \mathrm{mol}$ Trolox equivalent/L.

\section{Oxidative Stress Index}

The OSI was defined as the ratio of the TOS to TAS levels $(18,19)$. TAS levels were converted to $\mu \mathrm{mol}$. The percentage of TOS level to TAS level was regarded as the oxidative stress index (OSI). The serum OSI value was calculated as follows:

OSI $(\mathrm{AU})=\left[\left(\mathrm{TOS}, \mu \mathrm{mol} \mathrm{H}_{2} \mathrm{O}_{2}\right.\right.$ equivalent $\left./ \mathrm{L}\right) /(\mathrm{TAS}$, $\mu$ mol Trolox equivalent/L) $\times 100$. The results were expressed in Arbitrary Units.

\section{Statistical Analysis}

All statistical analyses were performed using SPSS for Windows version 17.0 (SPSS Inc., Chicago, IL, USA). Kolmogorov-Smirnov tests were used to test the normal- ity of data distribution. The data were expressed as arithmetic means and standard deviations. Independent sample T-test was respectively used in normally and non-normally distributed continuous variables between groups. Paired test was used to analyze changes within each group. Two-sided $\mathrm{p}$ value $<0.05$ was considered statistically significant.

\section{RESULTS}

The demographic, clinical and laboratory data of all study participants are shown in Table 1; no significant differences between the two groups regarding to these data were noted. All oxidative stress markers (TAS, TOS, and OSI) were significantly different among the groups ( $p<0.001$ for all) (Table 2). Compared to the control group, the TOS and OSI values of the IBS patients were determined to be higher and the TAS values were lower.

\section{DISCUSSION}

The main finding of the present study was that TOS values were higher and TAS values were lower in patients with IBS compared to control. In light of this information we can say there is an oxidative injury in the IBS although we cannot clear up whether it is a cause or a result.

Traditionally, IBS has been considered as a condition arising from brain-gut dysregulation and classified as one of the functional gastrointestinal disorders, hence its symptoms not explained by structural or biochemical abnormalities (4). Supports this fact IBS has been demonstrated accompanied by depression, anxiety, fibromyalgia syndrome and gastric esophagial reflux disease in resent studies (20-26).

Epidemiological studies have been shown the increased oxidative stress in inflammatory, degenerative and endocrine diseases which affect tissues integrity and metabolic rate of body. There were several studies demonstrating higher concentrations of oxidative stress products and antioxidants in the chronic inflammatory diseases of gastrointestinal tractus such as crohn disease, ulcerative colitis and chronic hepatitis. Especially superoxide dismutase protein carbonyl content (POPs), DNA oxidation products [8-hydroxy-2'-deoxyguanosine $(8-\mathrm{OHdG})]$, reactive oxygen intermediates (ROIs), lipid peroxidation products malonaldehyde (MDA) and 4-hydroxynonenal (HNE), catalase (CAT), glutathione peroxidase (GSH-Px), xanthine oxidase (XO), adenosine deaminase (AD) activities, and malondialdehyde (MDA) and nitric oxide (NO) were studied in these studies (27-31). However there is limited number of study evaluating oxidative stress products in IBS. Mete R. et al. (32) evaluated the plasma concentrations of malondialdehyde (MDA) and nitric oxide (NO) and the plasma activities of oxidant and antioxidant enzymes (Superoxide dismutase (SOD), catalase (CAT), glutathione peroxidase (GSH-Px), xanthine oxidase (XO), 
adenosine deaminase (AD) activities) patients with IBS. Plasma XO and AD activities, lipid peroxidation product MDA and NO concentrations were significantly higher in IBS patients than in controls. The SOD, CAT, and GSH-Px activities in the serum of patients with IBS were significantly lower than that of controls. Oran et al. (33) demonstrated the presence of oxidative stress, a disturbance in prooxidant - antioxidant balance and increased inflammation in patients with IBS by showing the drop in paraoxonase and arylesterase activities accompanied with an increase in conjugated diene levels. Urinary redox potential had also been studied in depression, MDA and SOD in fibromyalgia, which are the other functional diseases related with IBS (34-36). In our study, we evaluated the TAS and TOS values by Erel's method which has high linearity, rapid, easy, stable, reliable, sensitive, inexpensive and fully automated and the results are highly reproducible $(18,19)$. We found TOS was increased and TAS was decreased in patients with IBS compared to the controls similar to previous studies that specific oxidative stress markers were evaluated in.

Additionally it was tired to explain the oxidative stress plays a role in the pathogenesis of IBS by evaluating the potential effects of antioxidants in previous experimental studies. Firstly Asadi-Shahmirzadi et al. (37) demonstrated that the severity of stress-induced IBS was diminished by the Aloe vera/German chamomile mixture at all doses used but not dose-dependently, via inhibiting colonic MPO activity and improving oxidative stress status. Later Zhang et al. (38) evaluated the melatonin effects on gastric residual rate, small intestine propulsion rate and regeneration of gastric mucosa breaked down by noise stress. Melatonin is effective in reversing the gastrointestinal motility disorder and gastric stress ulcer on noise stress induced rats by its antioxidant and neuroendocrine activity in the circadian organization that previously demonstrated (38). Lastly Garabadu et al. (39) demonstrated that Eugenol protected against restraint stress induced development of IBS-like gastrointestinal dysfunction through modulation of HPA-axis and brain monoaminergic pathways apart from its antioxidant effect. Similar studies were performed in clinical subjects and demonstrated that antioxidants improves IBS symptoms. Kuiken SD et al. (40) argued a hypothesis that nitric oxide (NO) is involved in maintaining visceral hypersensitivity in IBS and NO synthase inhibitor NG -monomethyl-L-arginine (LNMMA) can restore on rectal resting volume, rectal sensitivity to distension and rectal compliance. Although L-NMMA did not alter rectal resting volume, rectal sensitivity to distension and rectal compliance, significantly increased the threshold for discomfort/pain in IBS patients (40). Melatonin is one of the favorite therapeutic agent plays an important role in gastrointestinal physiology and includes anxiolytic, anti-inflammatory and motility regulatory effects. In recent studies it was demons- trated that its antioxidant effects plays a role to improve IBS symptoms $(41,42)$.

In conclusion our present study demonstrated that there is increased oxidative stress and decreased antioxidant capacity in patient with IBS; therefore, we should consider that antioxidants might be beneficial in the supportive treatment of IBS. The limitation of the present study is that the TAS, TOS and OSI values were not examined after the IBS treatment and they were not compared to other inflammatory parameters and naturel antioxidants such as albumin bilirubin and uric ascide. To support our results prospective and randomized controlled trials are necessary.

Conflict of interest: None

\section{References}

1. WELLS NE, HAHN BA, WHORWELL PJ 1997 Clinical economics review: irritable bowel syndrome. Aliment Pharmacol Ther. 11: 1019-30. http://dx.doi.org/10.1046/j.1365-2036.1997.00262.x

2. GARAKANI A, WIN T, VIRK S, GUPTA S, KAPLAN D, MASAND PS 2003 Comorbidity of irritable bowel syndrome in psychiatric patients: a review. Am J Ther. 10: 61-7. http://dx.doi.org/10.1097/00045391-200301000-00014

3. TALLEY NJ, SPILLER R 2002 Irritable bowel syndrome: a little understood organic bowel disease? Lancet. 360: 555-64. http://dx.doi.org/10.1016/S0140-6736(02)09712-X

4. TALLEY NJ 2006 Irritable Bowel Syndrome. Intern Med J. 36: 724-8. http://dx.doi.org/10.1111/j.1445-5994.2006.01217.x

5. CROWELL MD 2004 Role of serotonin in the pathophysiology of the irritable bowel syndrome. Br J Pharmacol. 141: 1285-93. http://dx.doi.org/10.1038/sj.bjp.0705762

6. PICHE T, SAINT-PAUL MC, DAINESE R, MARINE-BARJOAN E, IANNELLI A, MONTOYA ML, PEYRON JF, CZERUCKA D, CHERIKH F, FILIPPI J, TRAN A, HÉBUTERNE X 2008 Mast cells and cellularity of the colonic mucosa correlated with fatigue and depression in irritable bowel syndrome. Gut. 57: 468-73. http://dx.doi.org/10.1136/gut.2007.127068

7. HEITKEMPER M, JARRETT M, CAIN K, SHAVER J, BOND E, WOODS NF, WALKER E 1996 Increased urine cathecolamines and cortisol in women with irritable bowel syndrome. Am J Gastroenterol. 91: 906-13.

8. COLUCCI R, BLANDIZZI C, BELLINI M, GHISU N, TONINI M, DEL TACCA M 2008 The genetics of the serotonin transporter and irritable bowel syndrome. Trends Mol Med. 14: 295304. http://dx.doi.org/10.1016/j.molmed.2008.05.001

9. Rome Foundation 2006 Guidelines_-Rome III Diagnostic Criteria for Functional Gastrointestinal Disorders. J Gastrointestin Liver Dis. 15(3): 307-12.

10. HALLIWELL B 1996 Antioxidants in human health and disease. Annu Rev Nutr. 16: 33-50. http://dx.doi.org/10.1146/annurev.nu.16.070196.000341

11. DUYGU F, KORUK ST, KARSEN H, AKSOY N, TASKIN A, HAMIDANOGLU M 2012 Prolidase and oxidative stress in chronic hepatitis C. J Clin Lab Anal. 26: 232-7. http://dx.doi.org/10.1002/jcla.21510

12. MORIYA K, NAKAGAWA K, SANTA T, SHINTANI Y, FUJIE H, MIYOSHI H, TSUTSUMI T, MIYAZAWA T, ISHIBASHI K, HORIE T, IMAI K, TODOROKI T, KIMURA S, KOIKE K 2001 Oxidative stress in the absence of inflammation in a mouse 
model for hepatitis $\mathrm{C}$ virus-associated hepatocarcinogenesis. Cancer Res. 61: 4365-70.

13. YALCIN S, ULAS T, EREN MA, AYDOGAN H, CAMUZCUOGLU A, KUCUK A, YUCE HH, DEMIR ME, VURAL M, AKSOY N 2013 Relationship between oxidative stress parameters and cystatin $\mathrm{C}$ levels in patients with severe preeclampsia. Medicina (Kaunas) 49: 118-23.

14. BOZKUS F, SAN I, ULAS T, IYNEN I, YESILOVA Y, GULER Y, AKSOY N 2013 Evaluation of total oxidative stress parameters in patients with nasal polyps. Acta Otorhinolaryngol Ital. 33(4): 248-53.

15. ULAS T, BUYUKHATIPOGLU H, KIRHAN I, DAL MS, ULAS S, DEMIR ME, EREN MA, UCAR M, HAZAR A, KURKCUOGLU IC, AKSOY N 2013 Evaluation of oxidative stress parameters and metabolic activities of nurses working day and night shifts. Rev Esc Enferm USP. 47: 471-6.

16. DEMIR M, ULAS T, TUTOGLU A, BOYACI A, KARAKAS EY, SEZEN H, USTUNEL M, BILINC H, GENCER M, BUYUKHATIPOGLU H 2014 Evaluation of oxidative stress parameters and urinary deoxypyridinoline levels in geriatric patients with osteoporosis. J Phys Ther Sci. 26: 1405-9.

http://dx.doi.org/10.1589/jpts.26.1405

17. ULAS T, BUYUKHATIPOGLU H, KIRHAN I, DAL MS, EREN MA, HAZAR A, DEMIR ME, AYDOGAN T, KARABABA F, UYANIKOGLU A, KURKCUOGLU IC 2012 The effect of day and night shifts on oxidative stress and anxiety symptoms of the nurses. Eur Rev Med Pharmacol Sci. 16: 594-9.

18. EREL O 2005 A new automated colorimetric method for measuring total oxidant status. Clin Biochem. 38: 1103-11. http://dx.doi.org/10.1016/j.clinbiochem.2005.08.008

19. EREL O 2004 A novel automated method to measure total antioxidant response against potent free radical reactions. Clin Biochem. 37: 112-9. http://dx.doi.org/10.1016/j.clinbiochem.2003.10.014

20. LYDIARD RB 2001 Irritable bowel syndrome, anxiety, and depression: what are the links? J Clin Psychiatry 62: 38-45.

21. DINAN TG, QUIGLEY EM, AHMED SM, SCULLY P, O'BRIEN S, O'MAHONY L, O'MAHONY S, SHANAHAN F, KEELING PW 2006 Hypothalamic-pituitary-gut axis dysregulation in irritable bowel syndrome: plasma cytokines as a potential biomarker? Gastroenterology. 130: 304-11.

http://dx.doi.org/10.1053/j.gastro.2005.11.033

22. FADGYAS-STANCULETE M, BUGA A-M, POPA-WAGNER A, DUMITRASCU DL 2014 The relationship between irritable bowel syndrome and psychiatric disorders: from molecular changes to clinical manifestations Mol Psychiatry. 2: 4.

http://dx.doi.org/10.1186/2049-9256-2-4

23. SLIM M, CALNDRE EP, RICO-VILLADEMOROS F 2015 An insight into the gastrointestinal component of fibromyalgia: clinical manifestations and potential underlying mechanisms. Rheumatol Int. 35: 433-44.

http://dx.doi.org/10.1007/s00296-014-3109-9

24. SPERBER AD., DEKEL R 2010 Irritable Bowel Syndrome and Co-morbid Gastrointestinal and Extra-gastrointestinal Functional Syndromes. J Neurogastroenterol Motil. 16(2): 113-9. http://dx.doi.org/10.5056/jnm.2010.16.2.113

25. KOUNTOURAS J1, CHATZOPOULOS D, ZAVOS C, BOURA P, VENIZELOS J, KALIS A 2002 Efficacy of trimebutine therapy in patients with gastroesophageal reflux disease and irritable bowel syndrome. Hepatogastroenterology. 49: 193-7.

26. MATHUR R, KO A, HWANG LJ, LOW K, AZZIZ R, PIMENTEL M 2010 Polycystic ovary syndrome is associated with an increased prevalence of irritable bowel syndrome. Dig Dis Sci. 55: 1085-9. http://dx.doi.org/10.1007/s10620-009-0890-5
27. LIH-BRODY L, POWELL SR, COLLIER KP, et. al. 1996 Increased oxidative stress and decreased antioxidant defenses in mucosa of inflammatory bowel disease. Dig Dis Sci. 41(10):2078-86. http://dx.doi.org/10.1007/BF02093613

28. D'ODORICO A, BORTOLAN S, CARDIN R, et. al. 2001 Reduced plasma antioxidant concentrations and increased oxidative DNA damage in inflammatory bowel disease. Scand J Gastroenterol. 36(12):1289-94. http://dx.doi.org/10.1080/003655201317097146

29. CHIARPOTTO E, SCAVAZZA A, LEONARDUZZI G et al. 1997 Oxidative damage and transforming growth factor beta 1 expression in pretumoral and tumoral lesions of human intestine. Free Radic Biol Med. 22(5): 889-94. http://dx.doi.org/10.1016/S0891-5849(96)00481-9

30. RANA SV, SHARMA S, PRASAD KK, SINHA SK, SINGH K 2014 Role of oxidative stress \& antioxidant defence in ulcerative colitis patients from north India. Indian J Med Res. 139(4): 568 71.

31. BARTSCH H, NAIR J 2005 Accumulation of lipid peroxidationderived DNA lesions: potential lead markers for chemoprevention of inflammation-driven malignancies. Mutat Res. 591(1-2): 34-44. http://dx.doi.org/10.1016/j.mrfmmm.2005.04.013

32. METE R, TULUBAS F, ORAN M, YILMAZ A, AVCI BA YILDIZ K et al 2013 The role of oxidants and reactive nitrogen species in irritable bowel syndrome: a potential etiological explanation. Med Sci Monit. 19: 762-6. http://dx.doi.org/10.12659/MSM.889068

33. ORAN M, TULUBAS F, METE R, AYDIN M, SARIKAYA HG, GUREL A 2014 Evaluation of paraoxonase and arylesterase activities in patients with irritable bowel syndrome. J Pak Med Assoc. 64: 820-2.

34. GRASES G, COLOM MA, FERNANDEZ RA, COSTABAUZÁ A, GRASES F 2014 Evidence of higher oxidative status in depression and anxiety. Oxid Med Cell Longev. 2014: 430216. http://dx.doi.org/10.1155/2014/430216

35. SALIM S 2014 Oxidative stress and psychological disorders. Curr Neuropharmacol. 12: 140-7. http://dx.doi.org/10.2174/1570159X11666131120230309

36. FATIMA G, DAS SK, MAHDI AA 2013 Oxidative stress and antioxidative parameters and metal ion content in patients with fibromyalgia syndrome: implications in the pathogenesis of the disease. Clin Exp Rheumatol. 31: S128-33.

37. ASADI-SHAHMIRZADI A, MOZAFFARI S, SANEI Y, BAEERI M, HAJIAGHAEE R, MONSEF-ESFAHANI HR, ABDOLLAHI M 2012 Benefit of Aloe vera and Matricaria recutita mixture in rat irritable bowel syndrome: Combination of antioxidant and spasmolytic effects. Chin J Integr Med. http://dx.doi.org/10.1007/s11655-012-1027-9

38. ZHANG L, GONG JT, ZHANG HQ, SONG QH, XU GH, CAI L, TANG XD, ZHANG HF, LIU FE, JIA ZS, ZHANG HW 2015 Melatonin Attenuates Noise Stress-induced Gastrointestinal Motility Disorder and Gastric Stress Ulcer: Role of Gastrointestinal Hormones and Oxidative Stress in Rats. J Neurogastroenterol Motil. 21: 189-99. http://dx.doi.org/10.5056/jnm14119

39. GARABADU D, SHAH A, SINGH S, KRISHNAMURTHY S 2015 Protective effect of eugenol against restraint stress-induced gastrointestinal dysfunction: Potential use in irritable bowel syndrome. Pharm Biol. 53: 968-74. http://dx.doi.org/10.3109/13880209.2014.950674

40. KUIKEN SD, KLOOKER TK, TYTGAT GN, LEI A, BOECKXSTAENS GE 2006 Possible role of nitric oxide in visceral hypersensitivity in patients with irritable bowel syndrome. Neurogastroenterol Motil. 18(2): 115-22. http://dx.doi.org/10.1111/j.1365-2982.2005.00731.x 
41. SIAH KTH, WONG RKM, HO KY 2014 Melatonin for the treatment of irritable bowel syndrome. World J Gastroenterol. 20(10): 2492-8. http://dx.doi.org/10.3748/wjg.v20.i10.2492
42. MOZAFFARI S, RAHIMI R, ABDOLLAHI M 2010 Implications of melatonin therapy in irritable bowel syndrome: a systematic review. Curr Pharm Des. 16(33): 3646-55.

http://dx.doi.org/10.2174/138161210794079254 